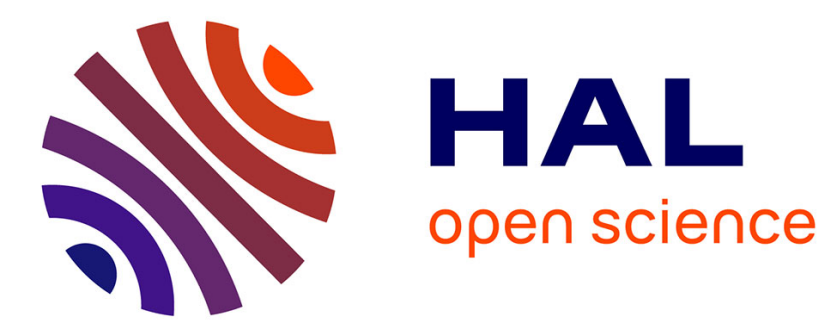

\title{
Asymmetric friction: Modelling and experiments
}

Nicolas Antoni, Jean-Louis Ligier, Philippe Saffré, Joseph Pastor

\section{To cite this version:}

Nicolas Antoni, Jean-Louis Ligier, Philippe Saffré, Joseph Pastor. Asymmetric friction: Modelling and experiments. International Journal of Engineering Science, 2007, 45 (45), pp.587-600. 10.1016/j.ijengsci.2007.04.014 . hal-00688732

\section{HAL Id: hal-00688732 \\ https://hal.science/hal-00688732}

Submitted on 26 Feb 2019

HAL is a multi-disciplinary open access archive for the deposit and dissemination of scientific research documents, whether they are published or not. The documents may come from teaching and research institutions in France or abroad, or from public or private research centers.
L'archive ouverte pluridisciplinaire HAL, est destinée au dépôt et à la diffusion de documents scientifiques de niveau recherche, publiés ou non, émanant des établissements d'enseignement et de recherche français ou étrangers, des laboratoires publics ou privés. 


\title{
Asymmetric friction: Modelling and experiments
}

\author{
N. Antoni ${ }^{\mathrm{b}}$, J.-L. Ligier ${ }^{\mathrm{a}, *}$, P. Saffré ${ }^{\mathrm{b}}$, J. Pastor ${ }^{\mathrm{b}}$ \\ ${ }^{\text {a }}$ RENAULT S.A., Powertrain Engineering Division, Rue des Bons Raisins, 92508 Rueil Malmaison, France \\ b LOCIE, MMSC Research Team, ESIGEC, 73376 Le Bourget du Lac, France
}

Received 20 June 2006; received in revised form 29 September 2006; accepted 18 October 2006

Available online 7 June 2007

\begin{abstract}
The effect of asymmetry with respect to sliding direction has been recently proved to be important in the analysis of static contact interactions of some automotive mechanical structures. In the present paper, we consider a nonclassical friction law, based on a dissymmetry of friction in a given sliding direction. A simple analytical micro-model has been adopted to derive the macroscopic model via averaging. Not only does it allow the law macroscopic characteristics to be determined but it also theoretically proves the existence of such dissymmetry of friction. The thermodynamic admissibility of the law is then shown from the derivation of a generalized standard model including friction. To complete the analysis, the existence of asymmetric friction conditions is confirmed from an experimental point of view. We then compare theoretical computations resulting from the analytical model with experimental results.
\end{abstract}

Keywords: Asymmetry; Friction; Micromechanics; Thermodynamics; Modelling; Experiments

\section{Introduction}

Some specific orthotropic surface textures are of interest in the automotive industry as they turned out to affect mechanical response at contacting surfaces by leading to dissymmetric behaviours (see e.g. [1,9]). A regularized asymmetric Coulomb-like law, characterized by a dissymmetry of friction with respect to sliding direction, has been used to reproduce some of these behaviours (see [1]). In this paper, we focus on the theoretical and experimental validity of this type of nonclassical friction law.

First, we introduce the static formulation. This is specific because it extends the classical Coulomb law of friction by including different friction coefficients depending on the path considered in a given principal sliding direction.

Due to the paucity of literature on this subject, it was necessary to prove the existence of such a friction dissymmetry theoretically. A micromechanical model has been adopted to derive this macroscopic property

\footnotetext{
* Corresponding author.

E-mail address: jean-louis.ligier@renault.com (J.-L. Ligier).
} 
by averaging. Indeed, various aspects of the micromechanics of friction are revelant in supporting the construction of nonclassical laws (see e.g. $[6,13,14]$ ). In this respect, we analyse an anisotropic friction model resulting from micromechanical considerations that determines the macroscopic parameters via averaging (see e.g. Mróz and Stupkiewicz [11] and Mróz [12]). This model also shows that the arbitrary assumptions previously used for the friction coefficients are realistic.

As a friction law may not be ordinary, we have then paid particular attention to demonstrating its thermodynamic admissibility. The concept of generalized standard materials adapted to frictional contact problems is used to rebuild a specific dual pseudo-potential so that the Clausius-Duhem inequality is satisfied (see e.g. Moreau [10], Felder [4,5] and Strömberg et al. [18]).

Second, the existence of such friction dissymmetry is proved from an experimental point of view. After describing experimental conditions, we present the main results obtained. A correlation with those resulting from the analytical model in equivalent conditions is also analysed.

\section{Theoretical aspects}

\subsection{A regularized asymmetric Coulomb-like law}

To include asymmetric frictional effects let us formally introduce a regularized asymmetric Coulomb-like law of friction. Such a law is assumed to hold pointwise on the contact interface and it takes two stages into account (see Fig. 1):

- A reversible stage corresponding to the elastic deformation of asperities at the contact interface: let $k_{t_{i}}$ represent the tangential contact stiffness, associated with the principal slip direction $\hat{t}_{i}(i=a, b)$, which is the typical parameter of this stage;

- An asymmetric irreversible stage corresponding to an asymmetric macroscopic slip: the value of the friction coefficient is different depending on the slip path direction considered. With this aim in view, we introduce arbitrary friction coefficients denoted as $\left(\mu_{a}^{+}, \mu_{a}^{-}\right)$and $\left(\mu_{b}^{+}, \mu_{b}^{-}\right)$, associated with the principal slip directions $\hat{t}_{a}$ and $\hat{t}_{b}$, respectively, according to the path direction considered. Their existence is the main point considered in this paper and will be proved in the next sections.

Let $\sigma_{n}$ be the normal stress at the contact interface. According to the two stages described above, the static formulation of this friction law is written as

$$
\left\{\begin{array}{l}
-\mu_{i}^{-}\left|\sigma_{n}\right|<\sigma_{t_{i}}<\mu_{i}^{+}\left|\sigma_{n}\right| \Rightarrow u_{t_{i}}=\sigma_{t_{i}} / k_{t_{i}} \\
\left\{\begin{array}{l}
\sigma_{t_{i}}=\mu_{i}^{+}\left|\sigma_{n}\right| \Rightarrow u_{t_{i}} \geqslant \sigma_{t_{i}} / k_{t_{i}} \\
\sigma_{t_{i}}=-\mu_{i}^{-}\left|\sigma_{n}\right| \Rightarrow u_{t_{i}} \leqslant \sigma_{t_{i}} / k_{t_{i}}
\end{array} \quad i=a, b\right.
\end{array}\right.
$$

where $\sigma_{t_{i}}$ and $u_{t_{i}}$ denote respectively the tangential stress and the tangential (relative) displacement associated with the principal slip direction $\hat{t}_{i}(i=a, b)$.

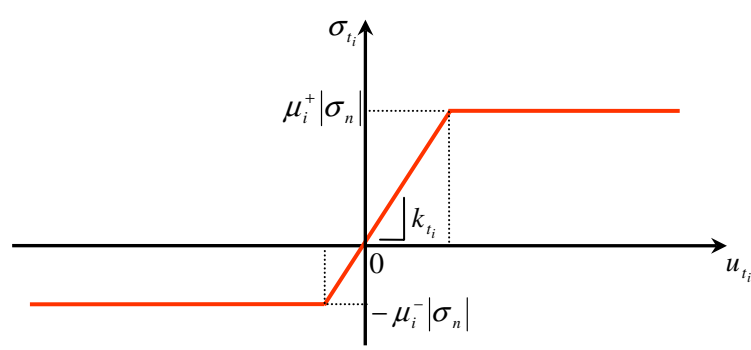

Fig. 1. A regularized asymmetric Coulomb-like law of friction. 


\subsection{Micromechanical approach of asymmetric friction}

Following Mróz [12], we consider the idealized surface texture (see Fig. 2a) of a fixed rigid body denoted as (2) on which long wedge-shaped asperities are periodically distributed (see Fig. 2b). Each of them is characterized by geometrical $(\alpha, \beta, h)$ as well as mechanical $\left(\mu_{\alpha}, \mu_{\beta}\right)$ properties. The parameter $\mu_{\alpha}$ (resp. $\left.\mu_{\beta}\right)$ represents the local friction coefficient along the wedge side denoted as $\left(C_{\alpha}\right)\left(\right.$ resp. $\left(C_{\beta}\right)$ ) (see Fig. $2 \mathrm{c}$ ).

Let us consider another rigid body denoted as (1) on which asperities are isotropically and uniformly distributed. These are modelled as identical elastic springs (i.e. the same original length, the same longitudinal stiffness $k_{n}$ and the same flexural stiffness $k_{f}$ ). Body (1) is kept in contact on body (2) under a prescribed vertical displacement $\delta \geqslant h$, sufficient to compress all springs (indeed the original spring length is assumed sufficiently greater than $h$ ), see Fig. 3 .

Let $\hat{t}_{a}$ denote a unit vector in the machining direction of the macroscopic horizontal contact plane (denoted as the nominal plane henceforth), $\hat{t}_{b}$ an orthogonal unit vector in the nominal contact plane and $\hat{n}$ the outward normal unit vector of body (2), thus defined as $\hat{n}=\hat{t}_{a} \wedge \hat{t}_{b}$. Then the steady macroscopic sliding of body (1) over body (2) is considered, assuming first an infinite flexural stiffness: the springs contract and release while sliding along wedge-shaped asperities so that the body (1) motion only occur in the nominal contact plane $\left(\hat{t}_{a}, \hat{t}_{b}\right)$.

The periodicity of both asperity distribution and loading on the body (2) surface texture allows an arbitrary period to be considered. As a consequence, the problem is reduced to the representative element (RE) depicted in Fig. 3.

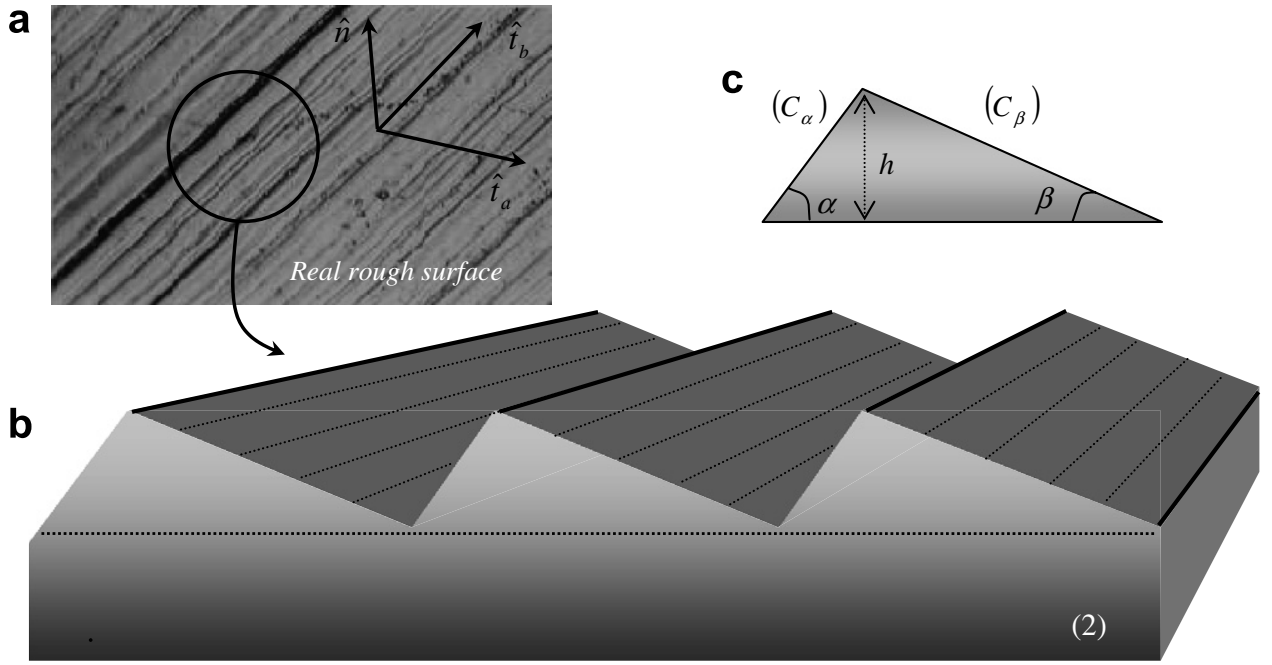

Fig. 2. A surface texture model.

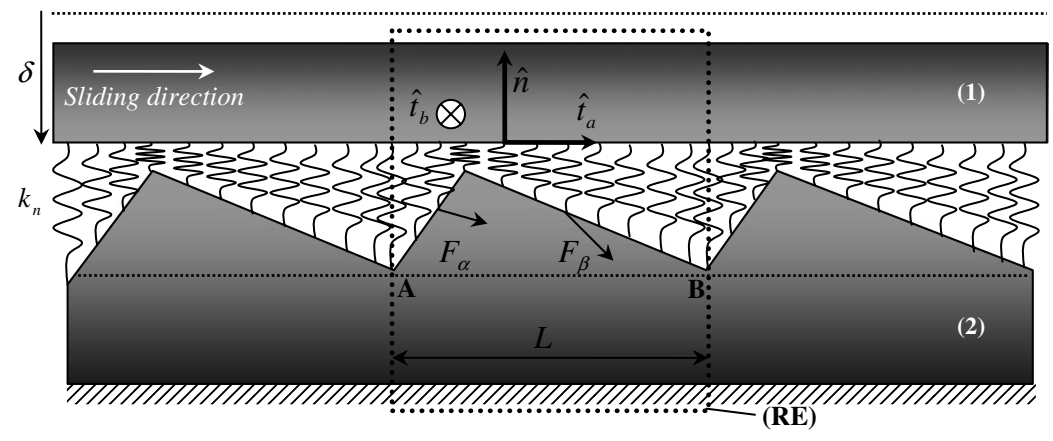

Fig. 3. A type of contact model. 
Let $\vec{F}_{\alpha}$ (resp. $\vec{F}_{\beta}$ ) be the real force (per unit of area of the real contact plane) applied by a spring on wedge $\left(C_{\alpha}\right)\left(\right.$ resp. $\left.\left(C_{\beta}\right)\right)$ during its slipping from point A to point B (see Fig. 3).

We thus define:

$$
\left\{\begin{array}{l}
\vec{F}_{\alpha}=F_{t_{a}}^{\alpha} \hat{t}_{a}+F_{n}^{\alpha} \hat{n} \\
\vec{F}_{\beta}=F_{t_{a}}^{\beta} \hat{t}_{a}+F_{n}^{\beta} \hat{n}
\end{array}\right.
$$

First, by assuming the classical Coulomb's law of friction remains valid for the microstructure, conditions for a spring end to slide along wedges $\left(C_{\alpha}\right)$ and $\left(C_{\beta}\right)$ are respectively written as

$$
\left\{\begin{array}{l}
F_{t_{a}}^{\alpha}=-\mu_{t_{a}}^{\alpha} F_{n}^{\alpha} \\
F_{t_{a}}^{\beta}=-\mu_{t_{a}}^{\beta} F_{n}^{\beta}
\end{array}\right.
$$

To ensure upward sliding of a spring along wedge $\left(C_{\alpha}\right)$, Eq. (3) must be associated with the following condition:

$$
\mu_{\alpha}<1 / \tan \alpha
$$

The adimensional parameters $\mu_{t_{a}}^{\alpha}$ and $\mu_{t_{a}}^{\beta}$ represent the apparent friction coefficients, respectively associated with wedges $\left(C_{\alpha}\right)$ and $\left(C_{\beta}\right)$, for the path direction previously defined, and they are given by:

$$
\left\{\begin{array}{l}
\mu_{t_{a}}^{\alpha}=H\left(\mu_{\alpha}, \alpha\right) \\
\mu_{t_{a}}^{\beta}=H\left(\mu_{\beta},-\beta\right)
\end{array}\right.
$$

where the function $H$ is defined as

$$
H(x, y)=\frac{x+\tan y}{1-x \tan y}
$$

Let $\left(u_{t_{a}}, u_{n}\right)$ be the components of a spring end displacement with respect to the $\left(\hat{t}_{a}, \hat{n}\right)$-basis.

The average value of the specific normal force (per unit of area of the nominal plane) applied by body (1) on body (2) is

$$
\bar{F}_{n}=\frac{1}{L}\left(\int_{0}^{h / \tan \alpha} F_{n}^{\alpha} \mathrm{d} u_{t_{a}}+\int_{h / \tan \alpha}^{L} F_{n}^{\beta} \mathrm{d} u_{t_{a}}\right)
$$

where $L=h\left(\frac{1}{\tan \alpha}+\frac{1}{\tan \beta}\right)$ is the period of wedge-shaped asperity distribution.

On the one hand, the contacting state of a spring end during its slipping along a wedge-shaped asperity provides the following kinematical relations:

$$
\left\{\begin{array} { l } 
{ u _ { t _ { a } } \in [ 0 , h / \operatorname { t a n } \alpha ] } \\
{ u _ { n } = u _ { t _ { a } } \operatorname { t a n } \alpha }
\end{array} \text { on } ( C _ { \alpha } ) \text { and } \left\{\begin{array}{l}
u_{t_{a}} \in[h / \tan \alpha, L] \\
u_{n}=h(1+\tan \beta / \tan \alpha)-u_{t_{a}} \tan \beta
\end{array} \text { on }\left(C_{\beta}\right)\right.\right.
$$

On the other hand, by assuming a linear elastic behaviour for springs of body (1), the average value of the specific normal force (per unit of area of the reference place) applied by body (1) on body (2) becomes

$$
F_{n}^{\alpha}=F_{n}^{\beta}=-\left(F_{0}+k_{n} u_{n}\right) \quad \forall u_{n} \in[0, h]
$$

where $F_{0}=-k_{n}(\delta-h) \leqslant 0$ is the real normal force applied by a spring on a wedge-shaped asperity at point A or B. By inserting Eq. (9) into Eq. (7) and taking relations (8) into account, it follows that

$$
\bar{F}_{n}=F_{0}-\frac{k_{n} h}{2}
$$

The average value of the specific tangential force (per unit of area of the nominal plane) applied by body (1) on body (2) is similarly defined as

$$
\bar{F}_{t_{a}}=\frac{1}{L}\left(\int_{0}^{h / \tan \alpha} F_{t_{a}}^{\alpha} \mathrm{d} u_{t_{a}}+\int_{h / \tan \alpha}^{L} F_{t_{a}}^{\beta} \mathrm{d} u_{t_{a}}\right)
$$


Let us now substitute real slip conditions expressed in Eq. (3) into the above definition. Next, by taking Eqs. (8) and (9) into account and comparing the result with Eq. (10), the average values of specific tangential and normal forces appear to be related by

$$
\bar{F}_{t_{a}}=-\mu_{a}^{+} \bar{F}_{n}
$$

From Eq. (12) we obtain the average value of specific friction coefficient in the principal direction $\hat{t}_{a}$, for the path direction considered:

$$
\mu_{a}^{+}=\frac{H\left(\mu_{\alpha}, \alpha\right) \tan \beta+H\left(\mu_{\beta},-\beta\right) \tan \alpha}{\tan \alpha+\tan \beta}
$$

It is worth noting that $\mu_{a}^{+}$is not dependent on $k_{n}$ and $h$.

We can also express the average value of specific friction coefficient in the principal direction $\hat{t}_{a}$, when the path direction is defined from point $\mathrm{B}$ to point $\mathrm{A}$, by reversing the parts of $\alpha$ and $\beta$ in the entire previous analysis which amounts to considering the following transformations:

$$
\left\{\begin{array} { l } 
{ \alpha \leftarrow \beta } \\
{ \beta \leftarrow \alpha }
\end{array} \text { and } \left\{\begin{array}{l}
\mu_{\alpha} \leftarrow \mu_{\beta} \\
\mu_{\beta} \leftarrow \mu_{\alpha}
\end{array}\right.\right.
$$

And, consequently:

$$
\mu_{a}^{-}=\frac{H\left(\mu_{\beta}, \beta\right) \tan \alpha+H\left(\mu_{\alpha},-\alpha\right) \tan \beta}{\tan \alpha+\tan \beta}
$$

where it has been similarly assumed that:

$$
\mu_{\beta}<1 / \tan \beta
$$

Eqs. (13) and (15) give asymmetric friction levels in the $\hat{t}_{a}$-direction:

$$
\mu_{a}^{+}-\mu_{a}^{-}=\left(\frac{2 \tan \alpha \tan \beta}{\tan \alpha+\tan \beta}\right) \frac{\left(\mu_{\alpha}^{2}-\mu_{\beta}^{2}\right)+\mu_{\alpha}^{2} \mu_{\beta}^{2}\left(\tan ^{2} \alpha-\tan ^{2} \beta\right)+\left(\mu_{\alpha}^{2} \tan ^{2} \alpha-\mu_{\beta}^{2} \tan ^{2} \beta\right)}{\left(1-\mu_{\alpha}^{2} \tan ^{2} \alpha\right)\left(1-\mu_{\beta}^{2} \tan ^{2} \beta\right)}
$$

It is consequently possible to reach a macroscopic friction asymmetry in the case of a complete asymmetry of the body (2) surface texture (geometrical and mechanical properties). Indeed, by taking sliding conditions (4) and (16) into account, it follows from Eq. (17) that:

$$
(\alpha \geqslant \beta) \text { and }\left(\mu_{\alpha} \geqslant \mu_{\beta}\right) \Rightarrow \mu_{a}^{+} \geqslant \mu_{a}^{-}
$$

This analysis thus shows that asymmetry in frictional response occurs for non-symmetric wedge asperity. This aspect was noted in [12] where only symmetric case $\left(\alpha=\beta, \mu_{\alpha}=\mu_{\beta}\right)$ was studied.

We shall calculate asymmetric friction levels $\mu_{a}^{+}-\mu_{a}^{-}$in the $\hat{t}_{a}$-direction for some profiles of a wedge-shaped asperity. Once mechanical $\left(\mu_{\alpha}, \mu_{\beta}\right)$ and geometric $(h / L)$ properties of the body $(2)$ surface texture are selected, we then find the admissible range of variations of $\alpha$ and $\beta$ to ensure satisfaction of sliding conditions (4) and (16). Asymmetric friction levels $\mu_{a}^{+}-\mu_{a}^{-}$in the $\hat{t}_{a}$-direction, together with the identically zero asymmetric friction level $\mu_{a}^{+}-\mu_{a}^{-}=0$ for comparison, are depicted in Fig. 4 , for symmetric $\left(\mu_{\alpha}=0.3, \mu_{\beta}=0.3\right)$ and asymmetric $\left(\mu_{\alpha}=0.3, \mu_{\beta}=0.2\right.$ and $\left.\mu_{\alpha}=0.2, \mu_{\beta}=0.3\right)$ cases. Note that Eq. (18) is graphically verified in Fig. 4.

As previously observed, Eq. (17) is independent on the height $h$ of wedge asperities. Consequently, it remains valid for a body (2) surface texture that is physically more realistic, with asperities of a constant shape (i.e. $(\alpha, \beta)$ constant) but with a Gaussian distribution of their heights (from the field, this is the most usual case but other distributions may also be considered, for instance Normal log. distribution). Note that the height $h$ for each wedge asperity must be measured from the same average line.

An infinite flexural stiffness has been assumed and thus a rigid body motion of the springs during the steady sliding of body (1). If this stiffness is assumed to be large but finite, there is then a bending of the springs prior to their sliding along wedge asperities in relation with a linear elastic behaviour which allows a reversible microslip $u_{t_{a}}^{\mathrm{e}}$ of body (1) relative to body (2) in the principal slip direction $\hat{t}_{a}$ of the nominal contact plane. 

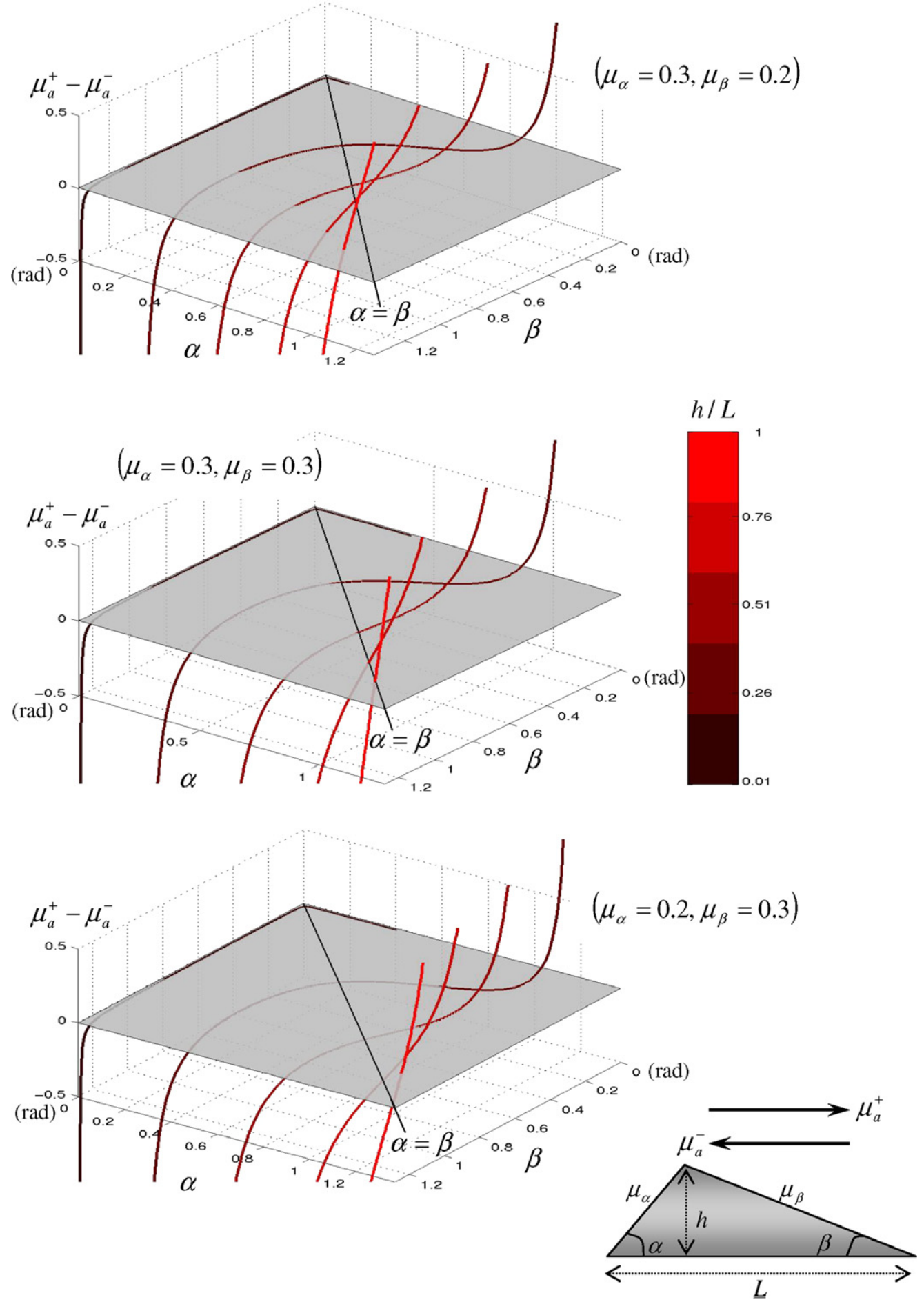

Fig. 4. Example of asymmetric friction levels in the $\hat{t}_{a}$-direction for some mechanical properties $\left(\mu_{\alpha}, \mu_{\beta}\right)$ of the body (2) surface texture.

The relation between principal tangential stress $\sigma_{t_{a}}$ at the nominal contact interface and this reversible displacement is thus linear, i.e.:

$$
u_{t_{a}}^{\mathrm{e}}=\frac{\sigma_{t_{a}}}{k_{t}}
$$


- On the one hand from a two-dimensional point of view, Eq. (19) can be generalized, assuming an isotropic bending behaviour of the springs, hence

$$
\vec{u}_{t}^{\mathrm{e}}=\frac{\vec{\sigma}_{t}}{k_{t}}
$$

- The springs start sliding in an elastically deformed state whenever the macro slip condition has been satisfied, which provides on the other hand a justification of the well-known decomposition (see, e.g. [2$5,7,10,18,19]$ ) of the total relative tangential displacement (observable) into one reversible elastic part denoted as $\vec{u}_{t}^{\text {e }}$ (however small it may be) and one irreversible inelastic part denoted as $\vec{u}_{t}^{\text {in }}$ and corresponding to the slipping:

$$
\vec{u}_{t}=\vec{u}_{t}^{\mathrm{e}}+\vec{u}_{t}^{\mathrm{in}}
$$

Eq. (20) is commonly adopted in the literature (see, e.g. Oden and Pires [13,14], Oden and Martins [15], Strömberg et al. [18] and Zhong [19]) but is not basically very realistic if any surface texture is taken into account. A reversible orthotropic phase could be therefore considered, characterized by two contact tangential stiffnesses $k_{t_{a}}$ and $k_{t_{b}}$ respectively associated with the principal slip directions $\hat{t}_{a}$ and $\hat{t}_{b}$.

\subsection{Thermodynamic admissibility}

A mechanical constitutive law must comply with certain principles to have a physical sense. In particular, it must be such that the two fundamental principles of thermodynamics, thus the Clausius-Duhem basic inequality, be satisfied.

Starting from experimental observations, a method based on the concept of generalized standard materials is applied in order to rebuild a law that is valid from a thermodynamic point of view. This approach is commonly used to derive mechanical constitutive models for material non-linearities (see, e.g. Lemaitre and Chaboche [8]).

Thus the general and natural framework for writing friction laws is the continuum thermodynamics. Since the method previously cited may not be directly particularized for the contact interface, it must therefore be adapted. An implementation has been proposed by several authors: Moreau [10] should be cited for his work on non-smooth phenomena such as plasticity and friction, as well as Felder [4,5] for his work on surface interactions applied to metal forming, and Strömberg et al. [18] for studying fretting (a wear process arising when contacting surfaces undergo small oscillatory displacements).

The general approach first consists in using the Clausius-Duhem inequality deduced from the expressions in local form of the first and second principles of thermodynamics at the contact interface. This inequality, which ensures the non-negativity of the dissipation denoted as $D_{C}$ at the contact interface, reads:

$$
D_{C}=D_{\text {Cintr }}+D_{C \text { th }} \geqslant 0
$$

where each part of the decomposition is defined as

$$
\left\{\begin{array}{l}
D_{\text {Cintr }}=\vec{\sigma}_{t} \cdot \dot{\vec{u}}_{t}-\left(\dot{\psi}_{C}+\dot{T}_{C} s_{C}\right) \\
D_{C \text { th }}=\sum_{k=1}^{2} \frac{\vec{q}_{k} \cdot \hat{n}_{k}}{T_{k}}\left(T_{k}-T_{C}\right)
\end{array}\right.
$$

$D_{\text {Cintr }}$ denotes the intrinsic (mechanical) dissipation. It actually represents the specific calorific power dissipated at the contact interface by physical and chemical mechanisms associated with friction. $D_{C \text { th }}$ denotes the thermal dissipation resulting from conductive heat transfer at the contact interface.

$\psi_{C}$ is the specific free energy for the area of the contact interface, $T_{C}$ is the intrinsic temperature on the contact interface, $s_{C}$ is the surface density of entropy on the contact interface, $\vec{q}_{k}$ and $T_{k}$ are the limits of the heat flux vector and the absolute temperature, respectively, when approaching the contact interface from within body $(k)$ and $\hat{n}_{k}$ is the outward unit normal vector on the contact interface. 
To demonstrate the thermodynamic admissibility of the regularized asymmetric Coulomb-like law of friction, in the frame of small displacements, let us consider the three following steps:

- The choice of a set of independent state variables, the internal representation of the state:

$$
\chi_{C}=\left(T_{C}, \vec{u}_{t}, \vec{u}_{t}^{\text {in }}, \chi_{C 3}, \ldots, \chi_{C n}\right)
$$

Let $Y=\left(Y_{C 0}, Y_{C 1}, Y_{C 2}, \ldots, Y_{C n}\right)$ be the variable vector in duality with the set of state variable rates denoted as $\dot{\chi}_{C}=\left(\dot{T}_{C}, \dot{\vec{u}}_{t}, \dot{\vec{u}}_{t}^{\text {in }}, \dot{\chi}_{C 3}, \ldots, \dot{\chi}_{C n}\right)$.

- The derivation of the state laws for reversible interactions from a specific free energy $\psi_{C}=\psi_{c}\left(\chi_{C}\right)$ :

$$
\left\{\begin{array}{l}
\vec{\sigma}_{t}=\frac{\partial \psi_{C}}{\partial \vec{u}_{t}}\left(\chi_{C}\right)=-\frac{\partial \psi_{C}}{\partial \vec{u}_{t}^{\text {in }}}\left(\chi_{C}\right) \\
s_{C}=-\frac{\partial \psi_{C}}{\partial T_{C}}\left(\chi_{C}\right)
\end{array}\right.
$$

The thermodynamic forces denoted as $\bar{Y}_{C}=\left(Y_{C 3}, \ldots, Y_{C n}\right)$ and respectively associated with the internal variables $\bar{\chi}_{C}=\left(\chi_{C 3}, \ldots, \chi_{C n}\right)$ are defined as

$$
\bar{Y}_{C}=-\frac{\partial \psi_{C}}{\partial \bar{\chi}_{C}}\left(\chi_{C}\right)
$$

- The derivation of the evolution laws for irreversible interactions from a specific dissipation potential denoted as $\Phi_{C}^{*}$, which must be taken as convex (but not necessarily differentiable) in order to ensure satisfaction of the dissipation inequality (22).

If $\Phi_{C}^{*}$ is differentiable:

$$
\dot{\bar{\chi}}_{C}=\frac{\partial \Phi_{C}^{*}}{\partial \bar{Y}_{C}}\left(\bar{Y}_{C}\right)
$$

If $\Phi_{C}^{*}$ is not differentiable everywhere:

$$
\dot{\bar{\chi}}_{C} \in \partial \Phi_{C}^{*}\left(\bar{Y}_{C}\right)
$$

where $\dot{\bar{\chi}}_{C}=\left(\dot{\vec{u}}_{t}^{\text {in }}, \dot{\chi}_{3}, \ldots, \dot{\chi}_{n}, \frac{\vec{q}_{1}, \hat{n}_{1}}{T_{1}}, \frac{\vec{q}_{2}, \hat{n}_{2}}{T_{2}}\right), \bar{Y}_{C}=\left(\vec{\sigma}_{t}, Y_{C 3}, \ldots, Y_{C n}, T_{1}-T_{C}, T_{2}-T_{C}\right)$ and $\partial \Phi_{C}^{*}\left(\bar{Y}_{C}\right)$ denotes the subdifferential of $\Phi_{C}^{*}$ with respect to $\bar{Y}_{C}$ (see Lemaitre and Chaboche [8], Moreau [10], Strömberg et al. [18]). Since the friction law considered here only takes purely mechanical mechanisms into account, the thermal aspect is removed. Thus, temperature should not appear in the following analysis.

\subsection{Set of independent state variables}

Let us consider the following elementary set:

$$
\chi_{C}=\left(\vec{u}_{t}, \vec{u}_{t}^{\mathrm{in}}\right)
$$

These two state variables (observable and internal, respectively) are assumed to comply with the decomposition in Eq. (21). The associated thermodynamic forces are respectively $Y_{C}=\left(\vec{\sigma}_{t}, \vec{\sigma}_{t}\right)$.

\subsection{Specific free energy and state laws}

The thermodynamic potential to be taken into account is written as

$$
\psi_{C}\left(\vec{u}_{t}, \vec{u}_{t}^{\mathrm{in}}\right)=\frac{1}{2} k_{t}\left(\vec{u}_{t}-\vec{u}_{t}^{\mathrm{in}}\right)^{2}
$$

Taking into account the decomposition in Eq. (20), the state law which derives from this thermodynamic potential is given by: 


$$
\vec{\sigma}_{t}=k_{t} \vec{u}_{t}^{\mathrm{e}}
$$

which is simply the reversible phase in Eq. (20).

\subsection{Specific dual pseudo-potential and evolution laws}

Let us consider the closed convex set $K \subset \boldsymbol{R}^{2}$ as follows:

$$
K=\left\{\vec{\sigma}_{t} \in \boldsymbol{R}^{2} / g\left(\vec{\sigma}_{t}, \sigma_{n}\right) \leqslant 0\right\}
$$

where $g$ must be a convex differentiable function, the so-called friction criterion.

The proposed dual pseudo-potential, from which the complementary laws derive, is defined as

$$
\Phi_{C}^{*}\left(\vec{\sigma}_{t}\right)=I_{K}\left(\vec{\sigma}_{t}\right)
$$

where $I_{K}$ denotes the indicator function of the set $K$ :

$$
I_{K}\left(\vec{\sigma}_{t}\right)= \begin{cases}0 & \text { if } \vec{\sigma}_{t} \in K \\ +\infty & \text { otherwise }\end{cases}
$$

Since $K$ is convex, $I_{K}$ is convex. This function is also minimum at $\vec{\sigma}_{t}=\overrightarrow{0}$.

Then the pseudo-potential $\Phi_{C}^{*}$ being convex with respect to the dual variable $\left(\vec{\sigma}_{t}\right)$ and minimum at $\left(\vec{\sigma}_{t}=\overrightarrow{0}\right)$, the Clausius-Duhem inequality is therefore satisfied.

The main difficulty is thus related to the determination of an appropriate expression of $g$. Several choices are clearly possible. The criterion expression we propose has been built by extending the usual orthotropic Coulomb friction limit criterion (see, e.g., Curnier [3], Felder [5], Mróz and Stupkiewicz [11], Mróz [12], Panagiotopoulos [17]). Considering the existence of asymmetric friction with respect to each principal slip direction, a dissymmetric friction limit criterion, differentiable and convex, is extrapolated as

$$
g\left(\sigma_{t_{a}}, \sigma_{t_{b}}, \sigma_{n}\right)=\left\{\begin{array}{l}
\left(\left(\frac{\sigma_{t_{a}}}{\mu_{a}^{+}}\right)^{2}+\left(\frac{\sigma_{t_{b}}}{\mu_{b}^{+}}\right)^{2}\right)^{1 / 2}-\left|\sigma_{n}\right| \quad \text { if } \sigma_{t_{a}} \geqslant 0 \text { et } \sigma_{t_{b}} \geqslant 0 \\
\left(\left(\frac{\sigma_{t_{a}}}{\mu_{a}^{-}}\right)^{2}+\left(\frac{\sigma_{t_{b}}}{\mu_{b}^{+}}\right)^{2}-\left|\sigma_{n}\right| \quad \text { if } \sigma_{t_{a}} \leqslant 0 \text { et } \sigma_{t_{b}} \geqslant 0\right. \\
\left(\left(\frac{\sigma_{t_{a}}}{\mu_{a}^{-}}\right)^{2}+\left(\frac{\sigma_{t_{b}}}{\mu_{b}^{-}}\right)^{2}\right)^{1 / 2}-\left|\sigma_{n}\right| \quad \text { if } \sigma_{t_{a}} \leqslant 0 \text { et } \sigma_{t_{b}} \leqslant 0 \\
\left(\left(\frac{\sigma_{t_{a}}}{\mu_{a}^{+}}\right)^{2}+\left(\frac{\sigma_{t_{b}}}{\mu_{b}^{-}}\right)^{2}\right)^{1 / 2}-\left|\sigma_{n}\right| \quad \text { if } \sigma_{t_{a}} \geqslant 0 \text { et } \sigma_{t_{b}} \leqslant 0
\end{array}\right.
$$

where $\left(\mu_{a}^{+}, \mu_{a}^{-}\right)$and $\left(\mu_{b}^{+}, \mu_{b}^{-}\right)$denote the friction coefficients associated with the principal slip directions $\hat{t}_{a}$ and $\hat{t}_{b}$, respectively, according to the path direction considered.

An example of representation $\left(\mu_{a}^{+}=0.2, \mu_{a}^{-}=0.08, \mu_{b}^{+}=0.12, \mu_{b}^{-}=0.15\right)$, together with that of a Coulomb isotropic friction criterion $\left(\mu_{a}^{+}=\mu_{a}^{-}=\mu_{b}^{+}=\mu_{b}^{-}=0.08\right)$ for comparison, is given in Fig. 5. It should be noted that the representation for this anisotropic and asymmetric criterion is made of four truncated ellipses.

Caution must be exercised on the physical interpretation of these last two formally entered friction coefficients. A write-up that is so general assumes the existence of a surface texture exhibiting such macroscopic friction properties, rarely observed in reality. The assumption of a single friction coefficient $\mu_{b}=\mu_{b}^{+}=\mu_{b}^{-}$ (generally different from the two other ones) associated with the principal direction $\hat{t}_{b}$ seems more realistic. However, such dissymmetry is theoretically possible and is taken here into account to give a general criterion form.

Denoting the component of the rate of the internal variable $\vec{u}_{t}^{\text {in }}$ in the $\hat{t}_{i}$-direction as $\dot{u}_{t_{i}}^{\text {in }}(i=a, b)$, the evolution law that derives from the convex (but not differentiable) dual pseudo-potential $\Phi_{C}^{*}$ is given by Eq. (28). It takes the following usual form (see Lemaitre and Chaboche [8], Moreau [10], Strömberg et al. [18]): 


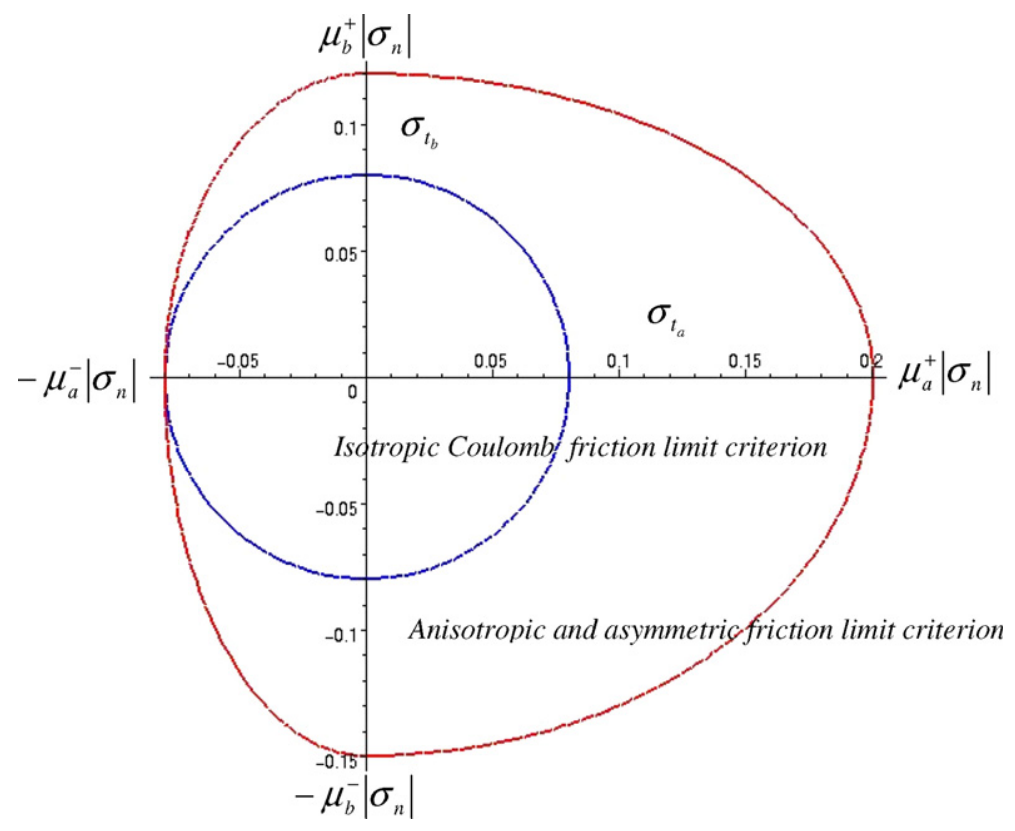

Fig. 5. Anisotropic and asymmetric friction limit criterion: example of representation.

$$
\dot{u}_{t_{i}}^{\text {in }}=\dot{\lambda} \frac{\partial g}{\partial \sigma_{t_{i}}}, \quad \dot{\lambda} \geqslant 0, \quad g \leqslant 0, \quad \dot{\lambda} g=0, \quad i=a, b
$$

A sliding rule, associated with the friction limit criterion, is thus assumed to hold. This is possible since a convex function $g$ has been selected here. However, the proposed friction limit criterion derives from the micromechanical model, only in the principal slip direction $\hat{t}_{a}$ where friction dissymmetry was demonstrated to exist.

The previous micromechanical analysis should entirely provide the limit friction criterion and the sliding rule, similarly as in Mróz and Stupkiewicz [11] and Mróz [12]. In this case, the concept of generalized standard materials is not valid since a non-associated sliding rule is generated from the wedge asperity model and there is no normality of the sliding vector to the friction surface in the contact plane.

The latter aspect is important and has to be experimentally verified. The presented experiments (Section 3) only point out friction asymmetry in principal direction $\hat{t}_{a}$ normal to the wedge line but do not provide any information on sliding vector orientation for the case of oblique orientation of wedge asperities with respect to applied load orientation.

The system (20), (21), (35) and (36) will then correspond to the complete formulation of this anisotropic and asymmetric friction model. Finally, it is easy to verify that such formulation in time rate-independent form and in the one-dimensional case is equivalent to that of the regularized asymmetric Coulomb-like law of friction (1).

\section{Comparison with experiments}

\subsection{Experimental conditions}

Two types of parallelepiped test specimens have been machined (see Fig. 6):

- The surface of the first, in steel material, presents identical wedge-shaped asperities (see Fig. 6a). The specified geometric parameters of each asperity are $\left(L=200 \mu \mathrm{m}, h=40 \mu \mathrm{m}, \alpha=45^{\circ}\right)$;

- The surface of the second, in aluminium, is characterized by a specified average height of asperities $(15 \mu \mathrm{m})$, see Fig. 6b. 
a

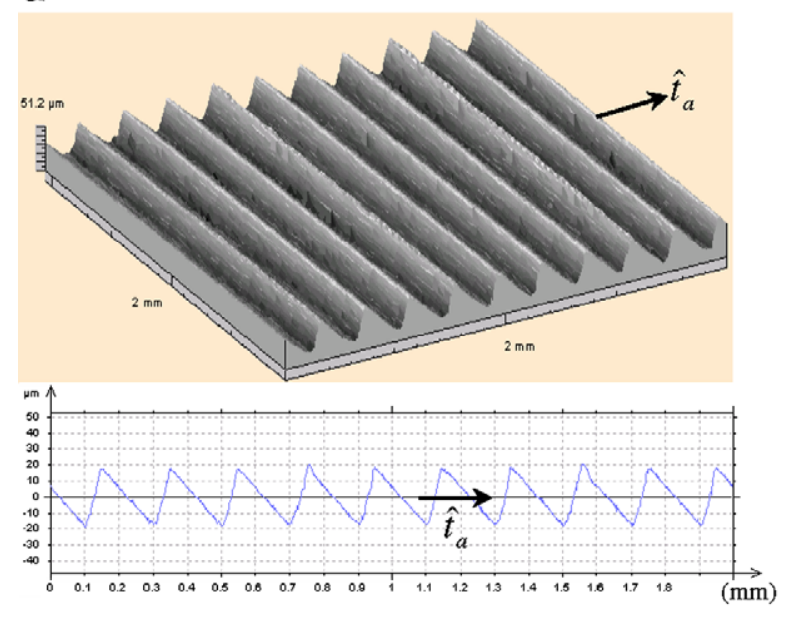

b

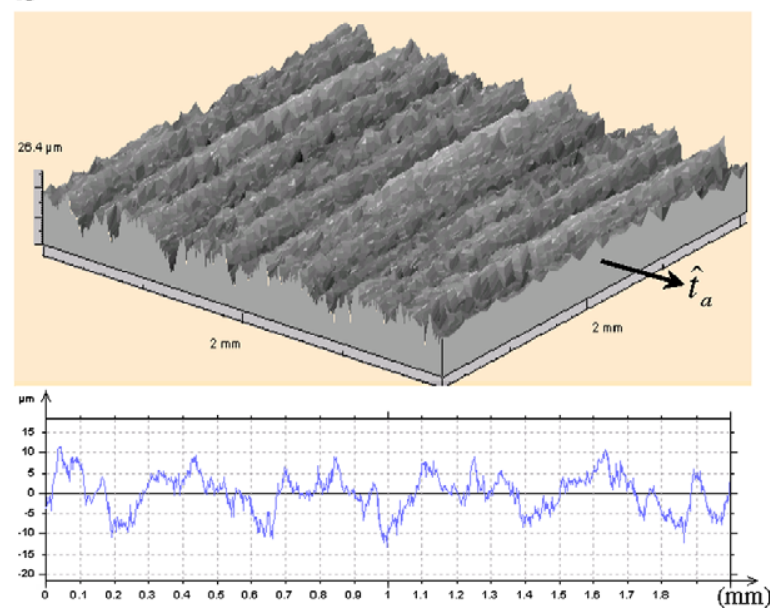

Fig. 6. Portions of test specimen functional surface textures: (a) Steel test specimen surface texture. (b) Aluminium test specimen surface texture.

Surface textures were checked (laser measurement) before the experiments. In particular, global geometric dispersion was proved to be very satisfactory, which is remarkable for a non standard machining of this type.

The typical friction test consists in keeping the two types of test specimens in contact under the application of a specified uniformly distributed normal load. The aluminium test specimen is then tangentially displaced in the machining direction $\hat{t}_{a}$ (of the nominal contact plane) on the fixed steel test specimen under the application of a tangential load, until steady macroscopic sliding is reached.

This is tested for both path directions $\left(+\hat{t}_{a}\right.$ and $-\hat{t}_{a}$, the conventions remain the same).

The aim of experiments is to observe and evaluate friction asymmetry in principal direction $\hat{t}_{a}$ normal to the wedge line.

An overview of experimental test bench, fully designed for this purpose, is given in Fig. 7. It is composed of the following parts:

- An hydraulic jack that applies the tangential load.

- A spring that applies a constant normal load.

- A rigid interface that ensures the application of a uniform contact pressure.

- An interface that ensures the perfect tangential guidance of the upper moving aluminium specimen test.

- Measuring cells that record normal and tangential load values and hydraulic jack displacement as a function of time. Note that the hydraulic jack tangential velocity is actually prescribed as a constant function $\left(0.5 \mathrm{~mm} \mathrm{~s}^{-1}\right)$.

\subsection{Experimental results and analysis}

We present here the test results obtained for a normal load intensity of $10^{3} \mathrm{~N}$. The variation of measured tangential load with aluminium test specimen displacement, according to the path direction considered in the principal slip direction $\hat{t}_{a}$, is depicted in Fig. 8 .

Note that these evolutions are given after functional surface lapping which corresponds to steady macroscopic sliding assumed for the previous micromechanical approach.

Thus in Fig. 8, we can observe that the values of tangential load tend to $330 \mathrm{~N}$ and $229 \mathrm{~N}$ for $+\hat{t}_{a}$ path and $-\hat{t}_{a}$ path directions, respectively. Consequently, the experimental corresponding friction coefficients are 0.33 and 0.29 , respectively

First, the friction test clearly shows the expected friction asymmetry since the coefficient of friction for $+\hat{t}_{a}$ path direction is greater than the one in the opposite path direction. 


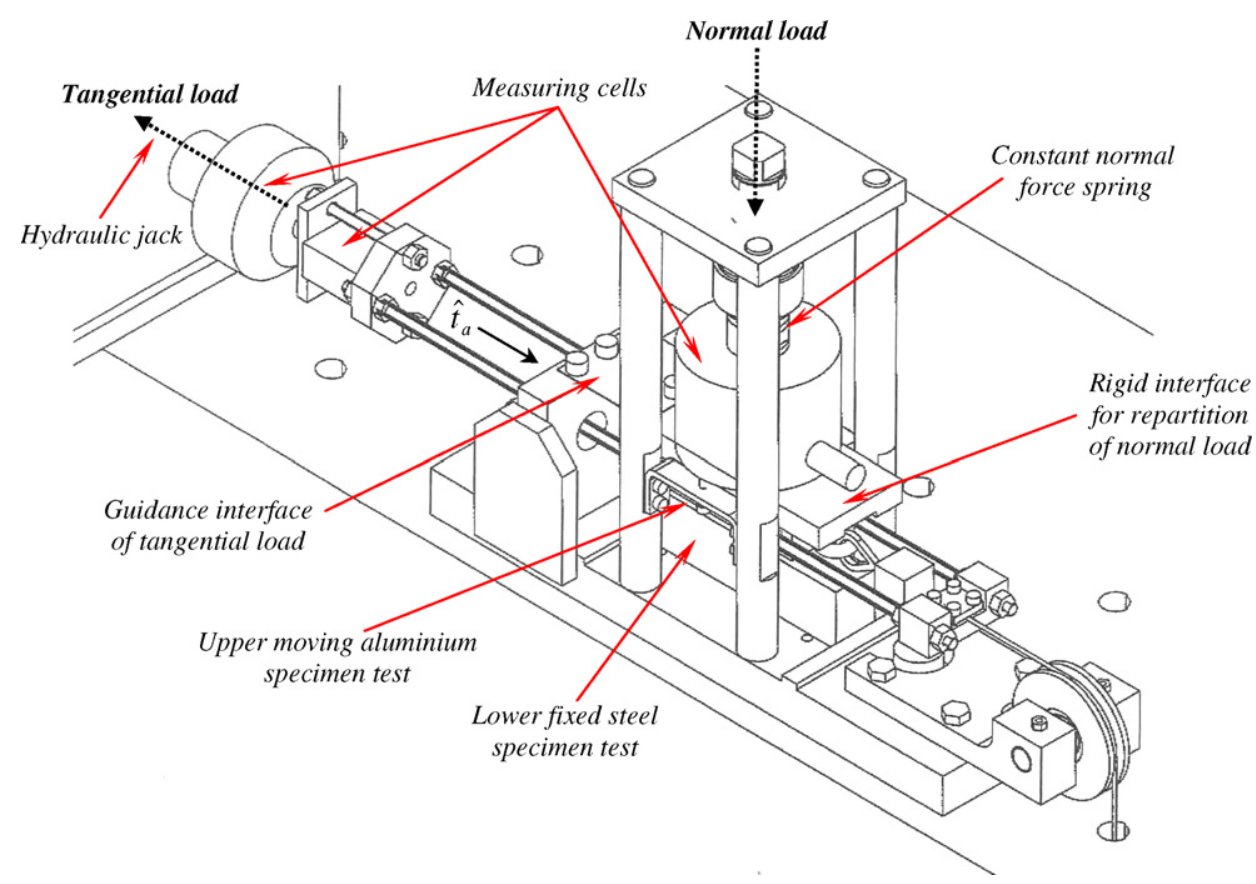

Fig. 7. Experimental test bench overview.

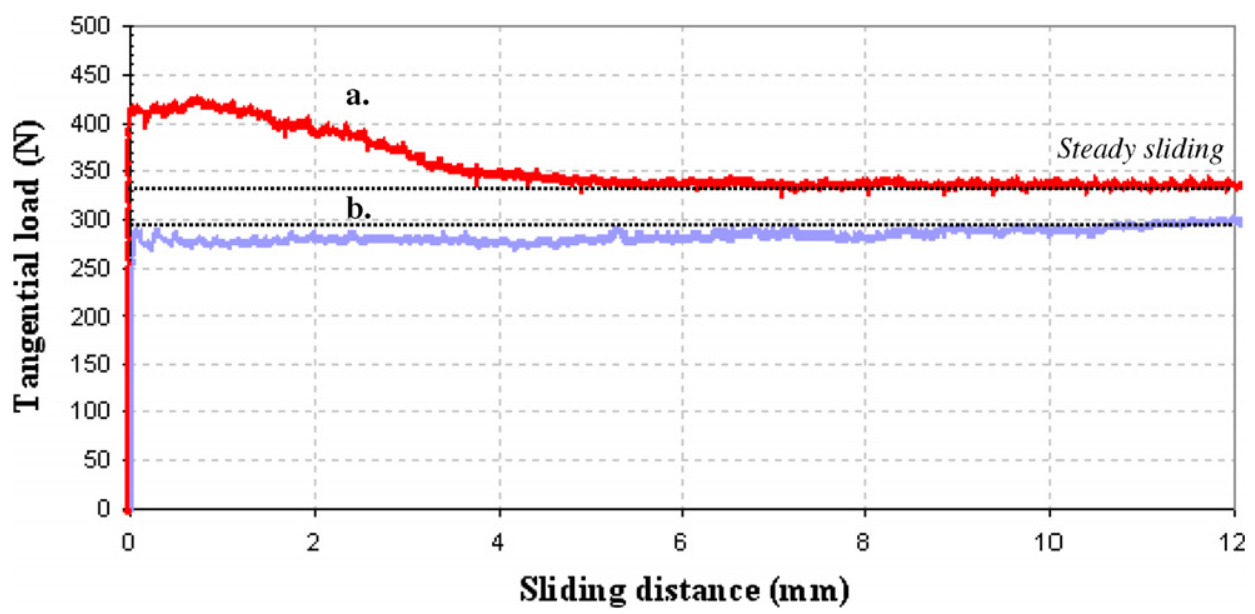

Fig. 8. Experimental variations in tangential load versus the aluminium test specimen displacement according to the path direction considered in the principal slip direction $\hat{t}_{a}$ : (a) $+\hat{t}_{a}$ path direction. (b) $-\hat{t}_{a}$ path direction.

Second, we can compare the values of measured friction coefficients with the computed values of the previous analytical model. The theoretical evolutions of friction coefficients as functions of the real coefficient of friction $\mu_{\alpha}=\mu_{\beta}=\mu$ (along a wedge-shaped asperity) are plotted in Fig. 9. They result from Eqs. (13) and (15). Although the real friction coefficient was not measured here, a reasonable admissible range of variations has been chosen $(0.23 \leqslant \mu \leqslant 0.29)$.

It appears for a real coefficient near 0.25 the calculation/test comparison is very good since calculated friction coefficients are $\mu_{a}^{+}=0.32$ and $\mu_{a}^{-}=0.29$, for $+\hat{t}_{a}$ path and $-\hat{t}_{a}$ path directions, respectively.

It is also important to note that sliding conditions (4) and (16) are well satisfied. Consequently, the use of the previous analytical model is admissible. 


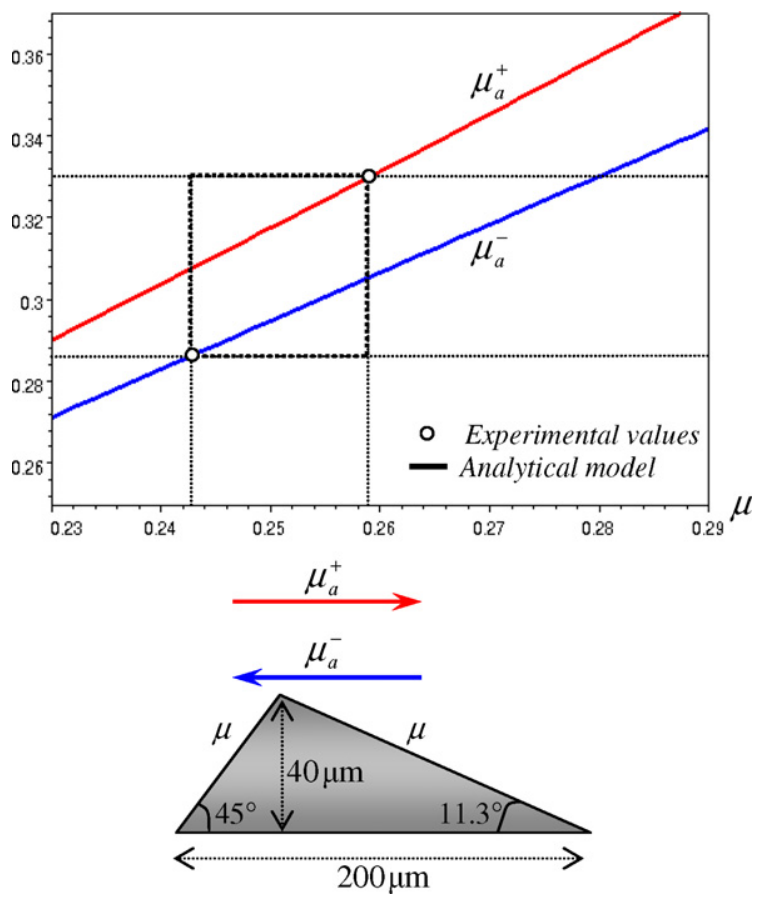

Fig. 9. Theoretical variations in macroscopic friction coefficients versus the real friction coefficient $\mu$, according to the path direction considered in the principal slip direction $\hat{t}_{a}$.

At this point, we would like to stress the general difficulty of parameter identification for a friction law, which has been achieved in this experimental study. The existence of a dissymmetry of friction has been proved from an experimental point of view for a given surface texture. Moreover, the calculation/experiments comparison has been proved to be very satisfactory.

\section{Conclusion}

Asymmetric friction conditions at the contact interface which characterize the proposed regularized asymmetric Coulomb-like law of friction have been achieved from micromechanical considerations on surface textures. This law, for which the thermodynamic validity has been proved by rebuilding a specific anisotropic and asymmetric friction limit criterion, is thus associated with a given surface texture. An analytical model has been proposed for evaluating macroscopic friction coefficients from micromechanical and geometric parameters of a type of surface texture.

In practice, we must underline that the available surface texture parameters in the industrial tools do not accurately enable the detection and characterization of surface textures of the type presented in this article. In order to validate this type of approach in spite of the simplifying assumptions used, experiments were conducted. On the one hand, the existence of asymmetric fiction has been successfully shown. On the other hand, experimental values of the friction coefficients were very similar to the ones computed with the analytical model.

Usual finite element methods do not allow the direct integration of a regularized asymmetric Coulomb-like law of friction. In this respect, various finite element procedures were also investigated and implemented using the proposed friction model for automotive mechanical structures.

\section{Acknowledgement}

This work is supported by RENAULT S.A.S. (Powertrain Engineering Division). 


\section{References}

[1] N. Antoni, Phénomène de microreptation des assemblages mécaniques, Thèse de l’Université de Savoie-Chambéry (France), Nov. 2005.

[2] J.-H. Cheng, N. Kikuchi, An incremental constitutive relation of unilateral contact friction for large deformation analysis, Journal of Applied Mechanics 52 (1985) 639-648.

[3] A. Curnier, A theory of friction, International Journal of Solids and Structures 20 (1984) 637.

[4] E. Felder, Formulation thermodynamique des interactions superficielles entre deux corps, Journal de Mécanique théorique et appliquée 4 (2) (1985) 283-303.

[5] E. Felder, Thermodynamics of surface interactions between two media with special emphasis on anisotropic effects, Journal de Mécanique théorique et appliquée 4 (2) (1992) 283-303.

[6] N. Kikuchi, J.T. Oden, Contact problems in elasticity: a study of variational inequalities and finite element methods, SIAM Studies in Applied Mathematics, Philadelphia, USA, 1988, pp. 274-280.

[7] A. Klarbring, Derivation and analysis of rate boundary-value problems of frictional contact, European Journal of Mechanics 9 (1990) 53-85.

[8] J. Lemaitre, J.-L. Chaboche, Mécanique des Matériaux Solides, 2eme Edition, Dunod, 1988, pp. $62-67$.

[9] J.-L. Ligier, E. Baron, Acyclisme et Vibrations, vol. 2, Analyses avancées et expérimentales, Editions Technip, 2002 , pp. 663-727.

[10] J.J. Moreau, Sur les Lois de Frottement, Plasticité et Viscosité, C.R. Académie des Sciences, Paris, t. 271, série A, 1970, pp. 608-611.

[11] Z. Mroz, S. Stupkiewicz, An anisotropic friction and wear model, Journal of Solid Structures 31 (1996) 1113-1131.

[12] Z. Mroz, Contact Friction Models and Stability Problems, CISM Courses and Lectures, vol. 457, Springer Wien, New York, 2002, pp. 179-232.

[13] J.T. Oden, E.B. Pires, Nonlocal and nonlinear friction laws and variational principles for contact problems in elasticity, Journal of Applied Mechanics 50 (1983) 67-73.

[14] J.T. Oden, E.B. Pires, Analysis of contact problems with friction under oscillating loads, Computer Methods in Applied Mechanics and Engineering 39 (1983) 337-362.

[15] J.T. Oden, J.A.C. Martins, Models and computational methods for dynamic friction phenomena, Computer Methods in Applied Mechanics and Engineering 52 (1985) 527-634.

[17] P.D. Panagiotopoulos, Hemivariational inequalities in frictional contact problems and applications, Studies in Applied Mechanics 11 (1985) 25-42.

[18] N. Strömberg, L. Johansson, A. Klarbring, Derivation and analysis of a generalized standard model for contact, friction and wear, International Journal of Solids and Structures 33 (13) (1996) 1816-1836.

[19] Z-H. Zhong, Finite Element Procedures for Contact-Impact Problems, Oxford University Press, 1993, pp. $136-167$. 